\title{
Detecting monopole charge in Weyl semimetals via quantum interference transport
}

\author{
Xin Dai, ${ }^{1}$ Hai-Zhou Lu, ${ }^{2, *}$ Shun-Qing Shen, ${ }^{3}$ and Hong Yao ${ }^{1,4, \dagger}$ \\ ${ }^{1}$ Institute for Advanced Study, Tsinghua University, Beijing 100084, China \\ ${ }^{2}$ Department of Physics, South University of Science and Technology of China, Shenzhen 518055, China \\ ${ }^{3}$ Department of Physics, The University of Hong Kong, Pokfulam Road, Hong Kong, China \\ ${ }^{4}$ Collaborative Innovation Center of Quantum Matter, Beijing 100084, China \\ (Received 22 December 2015; published 18 April 2016; corrected 29 April 2016)
}

\begin{abstract}
Topological Weyl semimetals can host Weyl nodes with monopole charges in momentum space. How to detect the signature of the monopole charges in quantum transport remains a challenging topic. Here, we reveal the connection between the parity of monopole charge in topological semimetals and the quantum interference corrections to the conductivity. We show that the parity of monopole charge determines the sign of the quantum interference correction, with odd and even parity yielding the weak antilocalization and weak localization effects, respectively. This is attributed to the Berry phase difference between time-reversed trajectories circulating the Fermi sphere that encloses the monopole charges. From standard Feynman diagram calculations, we further show that the weak-field magnetoconductivity at low temperatures is proportional to $+\sqrt{B}$ in double-Weyl semimetals and $-\sqrt{B}$ in single-Weyl semimetals, respectively, which could be verified experimentally.
\end{abstract}

DOI: 10.1103/PhysRevB.93.161110

Introduction. Topological Weyl semimetal is a new topological state of matter [1-15], in which the conduction and valence energy bands touch nontrivially at discrete momentum points, dubbed Weyl nodes. Remarkably, each Weyl node acts as a magnetic monopole in momentum space. Because the sum of monopole charges of all Weyl nodes in the Brillouin zone is zero, Weyl nodes must appear in pairs, namely the fermion-doubling theorem [2]. Within each pair, two Weyl nodes carry opposite monopole charges of $\mathcal{N}$ and $-\mathcal{N}$, respectively. Depending on $\mathcal{N}=1$ or 2 , the topological semimetals are referred to as single-Weyl semimetal and double-Weyl semimetal [5,16-18], respectively. The dispersion of the single-Weyl semimetal is linear in three dimensions, while the double-Weyl semimetal usually is linear in one and quadratic in the other two dimensions. Identifying the positions and monopole charges of Weyl nodes is crucial to qualitatively characterize a topological Weyl semimetal and understand the associated novel physical properties.

The monopole charge of a Weyl node also manifests as the number of stable surface Fermi arcs connecting paired Weyl nodes $[4,16]$, which can be directly measured in the angle-resolved photoemission spectroscopy (ARPES) experiments [19-23]. As an unambiguous measurement of the surface Fermi arcs in ARPES remains highly challenging, the quantum transport may provide an alternative approach to detect the signature of the monopole charges. For singleWeyl semimetals with $\mathcal{N}=1$, a $-\sqrt{B}$ dependence of the magnetoconductivity has been observed near zero magnetic field [24-32], which is one of the signatures for the weak antilocalization (WAL) effect in 3D systems [33]. The WAL effect arises from the quantum interference correction to the semiclassical conductivity. There have been increasing experimental efforts to search for topological Weyl semimetals with higher monopole charges such as $\mathcal{N}=2[5,17,18]$.

\footnotetext{
*Corresponding author: luhz@sustc.edu.cn

†Corresponding author: yaohong@tsinghua.edu.cn
}

Nevertheless, the quantum interference effects on the quantum transport phenomena caused by Weyl nodes with higher monopole charges remain unexplored.

In this Rapid Communication, we explicitly demonstrate the general relation between the monopole charge and quantum transport. We will focus on double-Weyl semimetals with $\mathcal{N}=2$ and single-Weyl semimetals with $\mathcal{N}=1$. Based on Feynman diagram calculations, we find that Weyl nodes with $\mathcal{N}=2$ in double-Weyl semimetals lead to a negative quantum interference correction to the conductivity compared to the positive correction in single-Weyl semimetals with $\mathcal{N}=1$. In double-Weyl semimetals, the monopole charge of $\mathcal{N}=2$ results in a $2 \pi$ Berry phase difference between two time reversed scattering loops. Such two loops can interfere constructively to enhance backscattering, leading to a weak localization (WL) correction in the quantum transport. While for single-Weyl semimetals, the phase difference is $\pi$, leading to destructive interference and hence the WAL effect. The qualitative difference in the quantum transport between single- and double-Weyl semimetals not only provides an experimental signature to identify monopole charges in momentum space but also forward our understanding to the quantum transport in 3D topological metals.

Weyl semimetals and monopole charges. The minimal model which can describe both single- and double-Weyl semimetals can be written as

$$
H=\left[\begin{array}{cc}
\chi v_{z} \hbar k_{z} & v_{\|}\left(\hbar k_{+}\right)^{\mathcal{N}} \\
v_{\|}\left(\hbar k_{-}\right)^{\mathcal{N}} & -\chi v_{z} \hbar k_{z}
\end{array}\right],
$$

where $k_{ \pm}=k_{x} \pm i k_{y}, \chi= \pm 1$ is the valley index, $v_{z}$ and $v_{\|}$are parameters and assumed to be constants, and momentum $\mathbf{k}$ is measured from the Weyl nodes. Here, $\mathcal{N}=1,2$ correspond to single- and double-Weyl semimetal, respectively. The model has a conduction band and a valence band, with the dispersions given by $\pm E_{\mathbf{k}}$ and $E_{\mathbf{k}}=\sqrt{v_{z}^{2} \hbar^{2} k_{z}^{2}+v_{\|}^{2}\left(\hbar^{2} k_{x}^{2}+\hbar^{2} k_{y}^{2}\right)^{\mathcal{N}}}$. Without loss of generality, we assume that the chemical potential is slightly above the Weyl nodes and the electronic transport is contributed mainly by the conduction bands throughout the 
paper. The eigenstate of the conduction band at valley $\chi=+$ is given by

$$
|\mathbf{k}\rangle=\left[\begin{array}{c}
\cos (\theta / 2) \\
\sin (\theta / 2) \exp (-i \mathcal{N} \varphi)
\end{array}\right],
$$

where $\cos \theta \equiv v_{z} k_{z} / E_{\mathbf{k}}$, and $\tan \varphi \equiv k_{y} / k_{x}$. The eigenstate of the conduction band around valley $\chi=-$ can be found by replacing $\cos (\theta / 2) \rightarrow \sin (\theta / 2)$ and $\sin (\theta / 2) \rightarrow-\cos (\theta / 2)$ in Eq. (2). The monopole charge can be found by integrating the Berry curvature over an arbitrary Fermi sphere $\Sigma$ that encloses the Weyl node,

$$
\frac{1}{2 \pi} \int_{\Sigma} d S \cdot \Omega= \pm \mathcal{N},
$$

with \pm for the \pm valleys, the Berry curvature [34] $\Omega=\nabla \times \mathbf{A}$, and $\mathbf{A}=\left(A_{\theta}, A_{\varphi}\right)$ is the Berry connection given by $A_{\theta}=$ $\left\langle\mathbf{k}\left|i \partial_{\theta}\right| \mathbf{k}\right\rangle=0$ and $A_{\varphi}=\left\langle\mathbf{k}\left|i \partial_{\varphi}\right| \mathbf{k}\right\rangle=\mathcal{N} \sin ^{2}(\theta / 2)$.

Monopole charge and quantum interference. In a disordered metal, the backscattering from a state with a wave vector of $\mathbf{k}$ to $-\mathbf{k}$ can be achieved by successive scattering via intermediate states. At sufficiently low temperatures, electrons can be scattered by many times but still maintain their phase coherence. In this quantum diffusive regime, the electric conductivity may acquire an extra correction from the quantum interference between the time-reversed scattering paths, leading to the WL or WAL effect [35]. We first focus on the Fermi sphere in one valley and assume no intervalley scattering. For each path [labeled as $P$ in Fig. 1(a)] connecting successive intermediate states of the backscattering from $\mathbf{k}$ to $-\mathbf{k}$ on the Fermi sphere, which encompasses the monopole charge at the origin, there exists a corresponding time-reversal counterpart $P^{\prime}$. The quantum interference is determined by the phase difference between the two time-reversed paths $P$ and $P^{\prime}$, which is equivalent to the Berry phase accumulated along the loop formed by $P$ together with $\bar{P} \equiv-P^{\prime}$, namely the corresponding path from $-\mathbf{k}$ to $\mathbf{k}$, as shown in Fig. 1(b).

The quantum interference correction then depends on the geometric phase, i.e., the Berry phase [34], collected by electrons after circulating the loop $\mathcal{C} \equiv P+\bar{P}$. The Berry phase can be found by a loop integral of the Berry connection

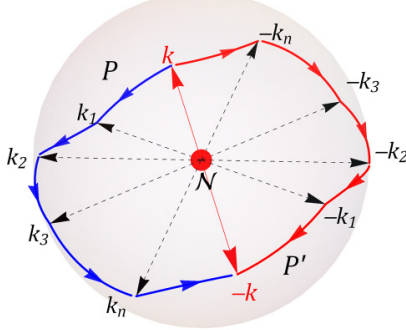

(a)

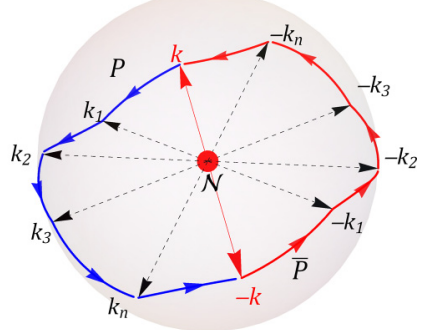

(b)
FIG. 1. The Fermi sphere in momentum space for a threedimensional topological semimetal, where the dot located at the origin represents a monopole charge of $\mathcal{N}$. (a) $P$ denotes a generic backscattering from the wave vector $\mathbf{k}$ to $-\mathbf{k}$ via intermediate states labeled as $\left(\mathbf{k}_{1}, \mathbf{k}_{2}, \ldots, \mathbf{k}_{n}\right) . P^{\prime}$ stands for the time-reversal counterpart of $P$. (b) The phase difference between $P$ and $P^{\prime}$ is equivalent to the Berry phase circulating around the loop $\mathcal{C}=P+\bar{P}$. around $\mathcal{C}$. Remarkably, this Berry phase depends only on the monopole charge, but not on the specific shape of the loop (see the rigorous proof in Ref. [36]):

$$
\gamma=\oint_{\mathcal{C}} d \boldsymbol{\ell} \cdot \mathbf{A}=\pi \mathcal{N}
$$

For double-Weyl semimetals, the monopole charge $\mathcal{N}=2$ and the Berry phase is then $2 \pi$. With the $2 \pi$ Berry phase, the time-reversed scattering loops interfere constructively, leading to the weak localization effect. However, for single-Weyl semimetals, the monopole charge is $\mathcal{N}=1$ and the Berry phase is $\pi$, which gives rise to the weak antilocalization effect. As the Berry phase is a consequence of the Berry curvature field generated by the monopole charge, we therefore establish a robust connection between the weak (anti)localization effect with the parity of monopole charge $\mathcal{N}$. The Berry phase argument is consistent with the symmetry classification [37], the single-Weyl semimetals belong to the symplectic class with a weak antilocalization correction, while double-Weyl semimetals correspond to the orthogonal class with a weak localization correction.

Feynman Diagram calculations. We now verify the above argument of quantum interference correction to conductivity in Weyl semimetals by the standard Feynman diagram calculations. The correction can be evaluated by calculating the maximally crossed diagrams, one of which is shown in Fig. 2. In this diagram, the segments of the arrow lines represent the intermediate states in the backscattering, and the dashed lines represent the correlation between the time-reversed scattering processes. The core calculation of the maximally crossed diagrams can be formulated into the particle-particle correlation, known as the cooperon. The cooperon of the double-Weyl semimetal is found to be (see Ref. [36] for details)

$$
\Gamma_{\mathbf{k}_{1}, \mathbf{k}_{2}} \approx \frac{\hbar}{2 \pi N_{F} \tau^{2}} \frac{e^{i 2\left(\varphi_{2}-\varphi_{1}\right)}}{D_{1}\left(q_{x}^{2}+q_{y}^{2}\right)+D_{2} q_{z}^{2}},
$$

where $\mathbf{q}=\mathbf{k}_{1}+\mathbf{k}_{2}$ is the cooperon wave vector, $\mathbf{k}_{1}$ and $\mathbf{k}_{2}$ are the wave vectors of incoming and outgoing states, respectively, $\varphi_{1}$ and $\varphi_{2}$ are the azimuth angles of corresponding wave vectors, $D_{1}=8 \tau E_{F} v_{\|} / 3 \pi$ and $D_{2}=\tau v_{z}^{2}$ are the diffusion coefficients, $N_{F}$ is the density of states, and $\tau$ is the momentum relaxation time. In contrast, the cooperon of the single-Weyl

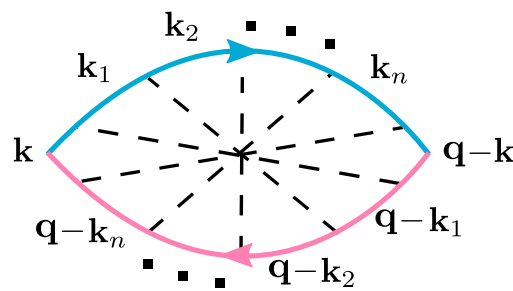

FIG. 2. The maximally crossed Feynman diagram that describes the quantum interference between the time-reversed scattering trajectories in Fig. 1 as $\mathbf{q} \rightarrow 0$. The arrowed solid and dashed lines denote the Green functions and impurity scattering, respectively. This kind of diagrams can give the quantum interference correction to the conductivity [38-40]. A negative (positive) correction corresponds to the weak (anti)localization effect, with the sign sensitive to the parity of the monopole charge. 
semimetal is known to take the form [33]

$$
\Gamma_{\mathbf{k}_{1}, \mathbf{k}_{2}} \approx \frac{\hbar}{2 \pi N_{F} \tau^{2}} \frac{1}{D q^{2}} e^{i\left(\varphi_{2}-\varphi_{1}\right)},
$$

where the diffusion coefficient $D=v_{F}^{2} \tau / 2$ [41]. Note that the main difference between Eqs. (5) and (6) lies in the phase factor involving $\varphi_{2}-\varphi_{1}$, which originates from different eigenstates of Weyl semimetals with different monopole charges.

As $\mathbf{q} \rightarrow 0$, i.e., $\mathbf{k}_{1}=-\mathbf{k}_{2}$, the cooperon becomes divergent and becomes the most dominant contribution to the backscattering. In this limit, $\varphi_{2}=\varphi_{1}+\pi$ [42]. Then, for the double-Weyl semimetal,

$$
\Gamma_{\mathbf{k}, \mathbf{q}-\mathbf{k}} \approx+\frac{\hbar}{2 \pi N_{F} \tau^{2}} \frac{1}{D_{1}\left(q_{x}^{2}+q_{y}^{2}\right)+D_{2} q_{z}^{2}},
$$

and for the single-Weyl semimetal,

$$
\Gamma_{\mathbf{k}, \mathbf{q}-\mathbf{k}} \approx-\frac{\hbar}{2 \pi N_{F} \tau^{2}} \frac{1}{D q^{2}}
$$

Note the different signs in Eqs. (7) and (8), which correspond to the WL and WAL effects, respectively. This is a direct consequence of different phase factors in the wave functions, generated by different monopole charges in double- and singleWeyl semimetals. In other words, a connection is therefore firmly established between the parity of monopole charge $\mathcal{N}$ and the sign of the quantum interference correction, with odd and even parity giving rise to WAL and WL, respectively. This is the main result of the paper.

Weak-localization conductivity. From the obtained cooperon above, it is straightforward to compute the quantum interference corrections to the Drude conductivity (see Ref. [36] for details). For double-Weyl semimetals, the quantum interference corrections to the Drude conductivity are intrinsically anisotropic because of the anisotropic dispersions in double-Weyl semimetals

$$
\begin{aligned}
\sigma_{z z}^{\mathrm{qi}} & =-\frac{9 e^{2}}{4 \pi^{2} \hbar} \frac{v_{z}}{2} \sqrt{\frac{1}{2 v_{\|}} \frac{1}{\pi E_{F}}}\left(\frac{1}{\ell_{z}}-\frac{1}{\ell_{\phi}}\right), \\
\sigma_{x x}^{\mathrm{qi}} & =-\frac{e^{2}}{4 \pi^{2} \hbar} \frac{2}{v_{z}} \sqrt{2 v_{\|} \pi E_{F}}\left(\frac{1}{\ell_{x}}-\frac{1}{\ell_{\phi}}\right),
\end{aligned}
$$

where $\ell_{i}$ is the mean free path and $\ell_{\phi}$ is the phase coherence length. The mean free path $\ell_{i}$ is defined as $\ell_{i}=\sqrt{D_{i} \tau}$. In the quantum diffusive regime, $\ell_{i}$ is much shorter than $\ell_{\phi}$, so the correction is negative in all directions. This negative correction is one of the signatures of the WL effect, consistent with the above Berry-phase argument of monopole charge. Note that this negative correction due to the WL effect is not divergent, as expected in 3D. Moreover, the total conductivity $\sigma=\sigma^{\mathrm{sc}}+\sigma^{\mathrm{qi}}$, where $\sigma^{\mathrm{sc}}$ is the semiclassical Drude conductivity, is still finite. From $\ell_{i}=v_{i} \tau$ and $\tau \propto$ $1 / E_{F}$, we obtain that $\sigma_{z z}^{\mathrm{qi}} \propto-E_{F}^{1 / 2}$ and $\sigma_{x x}^{\mathrm{qi}} \propto-E_{F}^{3 / 2}$ at low temperatures. The semiclassical conductivity is found to be $\sigma_{z z}^{\mathrm{sc}}=e^{2} N_{F} v_{z}^{2} \tau \propto E_{F}^{0}$ and $\sigma_{x x}^{\mathrm{sc}}=(8 / 3 \pi) e^{2} \tau N_{F} E_{F} v_{\|} \propto E_{F}$, where the density of states at the Fermi level per valley is $N_{F}=E_{F} / 8 \pi \hbar^{3} v_{z} v_{\|}$.

Weak localization induced magnetoconductivity. The negative quantum interference correction in Eq. (9) can be destroyed by a small magnetic field, giving rise to a positive magnetoconductivity as another signature of the weak localization in double-Weyl semimetals. The magnetoconductivity is anisotropic, depending on the field direction. When the field is applied along the $z$ direction, the quantum interference correction of the conductivity is found as

$$
\begin{aligned}
\sigma_{z z}^{\mathrm{qi}}(B)= & -\frac{3 e^{2} \tau v_{z}^{2}}{8 \hbar \pi^{2}} \int_{0}^{1 / \ell_{z}} d q_{z} \\
& \times\left[\psi\left(\frac{\ell_{B}^{2}}{\ell_{z}^{2}}+\ell_{B}^{2} q_{z}^{2}+\frac{1}{2}\right)-\psi\left(\frac{\ell_{B}^{2}}{\ell_{\phi}^{2}}+\ell_{B}^{2} q_{z}^{2}+\frac{1}{2}\right)\right],
\end{aligned}
$$

where $\psi$ is the digamma function and $\ell_{B} \equiv \sqrt{\hbar / 4 e D_{1} B}$ is the effective magnetic length in the $z$ direction. When the field is applied in the $x-y$ plane, the conductivity is given by the same formula as Eq. (10) but with $\ell_{B}^{2}=\hbar / 4 e B \sqrt{D_{1} D_{2}}$. The magnetoconductivity is defined as $\delta \sigma_{z z}^{\mathrm{qi}}(B)=\sigma_{z z}^{\mathrm{qi}}(B)-\sigma_{z z}^{\mathrm{qi}}(0)$. In the limit of $\ell_{\phi} \gg \ell_{B} \gg \ell_{z}$, which can be approached at low temperatures, the magnetoconductivity $\delta \sigma_{z z}^{\mathrm{qi}}(B) \propto \sqrt{B}$. In the limit of $\ell_{B} \gg \ell_{\phi}$ and $\ell_{B} \gg \ell_{z}, \delta \sigma_{z z}^{\mathrm{qi}}(B) \propto B^{2}$. As a good approximation, the weak localization induced magnetoconductivity in double-Weyl semimetals can be fitted as

$$
\delta \sigma_{z z}^{\mathrm{qi}}=C_{1}^{\mathrm{qi}} \frac{B^{2} \sqrt{B}}{B_{c}^{2}+B^{2}}+C_{2}^{\mathrm{qi}} \frac{B_{c}^{2} B^{2}}{B_{c}^{2}+B^{2}},
$$

where the fitting parameters $C_{1}^{\mathrm{qi}}$ and $C_{2}^{\mathrm{qi}}$ are positive and the critical field $B_{c}$ is related to the phase coherence length $\ell_{\phi}$ according to $B_{c} \sim \hbar / e \ell_{\phi}^{2}$. Empirically, the phase coherence length becomes longer with decreasing temperature and can be written as $\ell_{\phi} \sim T^{-p / 2}$; then $B_{c} \sim T^{p}$, where $p$ is positive and determined by decoherence mechanisms such as electronelectron interaction ( $p=3 / 2$ ) or electron-phonon interaction $(p=3)$. At high temperatures, $\ell_{\phi} \rightarrow 0$; thus, $B_{c} \rightarrow \infty$ and we have $\delta \sigma_{z z}^{\text {qi }} \propto B^{2}$. At low temperatures, $\ell_{\phi} \rightarrow \infty$; then $B_{c}=0$ and we have $\delta \sigma_{z z}^{\mathrm{qi}} \propto \sqrt{B}$.

Chiral-anomaly induced magnetoconductivity. Another mechanism that can also contribute a positive magnetoconductivity is the chiral anomaly. The nontrivial momentum-space Berry curvature in Weyl semimetals can induce an anomalous velocity which can couple with external magnetic fields [34], leading to a positive magnetoconductivity when the electric current is in parallel with magnetic fields $[43,44]$. In singleWeyl semimetals at weak fields, this "chiral anomaly" induced magnetoconductivity is found to be proportional to $B^{2}[43,44]$ or $B$ [45], depending on the Fermi energy. As a semiclassical effect, the chiral anomaly is not as sensitive to phase coherence and temperature as the weak localization effect is, so a similar positive magnetoconductivity is also expected in double-Weyl semimetals, without sign reversing, although the monopole charge is doubled. In addition, the chiral anomaly has a strong angle dependence, becomes prominent only in parallel magnetic fields. In contrast, the weak localization happens in all directions. These properties distinguish the positive magnetoconductivity of the chiral anomaly from that of the weak localization effect. In perpendicular magnetic fields, the Lorentz force gives rise to the classical negative magnetoconductivity that is proportional to $-\mu^{2} B^{2}$, where the mobility $\mu=2 e D / E_{F}$ in double-Weyl semimetals. Because of the 
TABLE I. Comparison between 3D single- and double-Weyl semimetals. $\pm E_{\mathbf{k}}$ is the dispersion relation of the conduction and valence bands, $N(E)$ is the density of states; $\delta \sigma^{q i}(B)$ is the weak (anti)localization magnetoconductivity when $\ell_{\phi} \gg \ell_{B} . \pm \mathcal{N}$ is the monopole charge.

\begin{tabular}{ccc}
\hline \hline & 3D single-Weyl & 3D double-Weyl \\
\hline$E_{\mathbf{k}}$ & $\hbar \sqrt{v_{\|}^{2}\left(k_{x}^{2}+k_{y}^{2}\right)+v_{z}^{2} k_{z}^{2}}$ & $\hbar \sqrt{v_{\|}^{2} \hbar^{2}\left(k_{x}^{2}+k_{y}^{2}\right)^{2}+v_{z}^{2} k_{z}^{2}}$ \\
$N(E)$ & $E^{2} / 2 \pi^{2} v_{\|}^{2} v_{z} \hbar^{3}$ & $E / 8 \pi v_{z} v_{\|} \hbar^{3}$ \\
$\delta \sigma^{q i}(B)$ & $-\sqrt{B}$ & $\sqrt{B}$ \\
$\mathcal{N}$ & 1 & 2 \\
\hline
\end{tabular}

functional relations, at sufficiently low temperatures the $\mathrm{WL}$ effect induced $\sqrt{B}$ magnetoconductivity always overwhelms the magnetoconductivity from the chiral anomaly and Lorentz force. For a comparison between single- and double-Weyl semimetals, we summarize the key results in Table I.

Effects of intervalley scattering. For single-Weyl semimetals, in the limit where intervalley scattering dominates, the quantum interference correction can be negative, i.e., a crossover to the weak localization [33], which is a result of the cancellation of $\pm \pi$ Berry phase when circulating monopoles with opposite charges. Similar phenomena were also studied in 2D graphene [40,46-48]. However, in doubleWeyl semimetals, even if intervalley scattering is considered where the electron scattering trajectory encloses the nodes of double-Weyl semimetals with opposite monopole charges, the weak localization is still robust since the total phase acquired by circulating the two monopoles is still an integer multiple of $2 \pi$. The crossover can be also understood from the symmetry classification [37], with $\pm \pi( \pm 2 \pi)$ Berry phase corresponding to the symplectic (orthogonal) class. In the limit of weak intervalley scattering, the qualitatively different quantum interference corrections can distinguish the Weyl nodes with different monopole charges.

Conclusions and discussions. To summarize, we have presented an intuitive picture revealing the connection between quantum interference correction to the conductivity and monopole charge in three-dimensional Weyl semimetals. The picture is verified by standard Feynman diagram calculations. It explains the weak antilocalization observed in single-Weyl semimetals [24-27] and predicts the weak localization in double-Weyl semimetals. Experimentally, the quantum interference correction can manifest through magnetoconductivity. In the zero temperature limit, the magnetoconductivity $\propto \mp \sqrt{B}$ for single- and double-Weyl semimetals, respectively. So far, there have been several materials, e.g., $\mathrm{HgCr}_{2} \mathrm{Se}_{4}[5,16]$ and $\mathrm{SrSi}_{2}$ [18] proposed as candidates for double-Weyl semimetals.

It is worthwhile to mention that, if weak higher order terms which may break the time-reversal symmetry are included in the $\mathbf{k} \cdot \mathbf{p}$ Hamiltonian, the quantum interference correction will be slightly smaller, as pointed out in Ref. [48]. Even though interactions can also contribute to conductivity, interactioninduced magnetoconductivity usually has much weaker magnetic field dependence compared to the one induced by the quantum interference $[33,35,49]$; it is expected that our results above are robust if weak interactions are included.

Acknowledgments. We would like to thank $\mathrm{Zi}$-Xiang $\mathrm{Li}$ and Shao-Kai Jian for stimulating discussions. This work was supported by the NSFC under Grant No. 11474175 (X.D. and H.Y.) and 11574127 (H.-Z.L.), the National Thousand-YoungTalents Program (H.-Z.L. and H.Y.), and by the Research Grant Council, University Grants Committee, Hong Kong under Grant No. 17303714.
[1] H. Weyl, Z. Phys. 56, 330 (1929).

[2] H. B. Nielsen and M. Ninomiya, Phys. Lett. B 130, 389 (1983).

[3] G. E. Volovik, The Universe in a Helium Droplet (Oxford University Press, Oxford, 2009).

[4] X. Wan, A. M. Turner, A. Vishwanath, and S. Y. Savrasov, Phys. Rev. B 83, 205101 (2011).

[5] G. Xu, H. M. Weng, Z. J. Wang, X. Dai, and Z. Fang, Phys. Rev. Lett. 107, 186806 (2011).

[6] A. A. Burkov and L. Balents, Phys. Rev. Lett. 107, 127205 (2011).

[7] K. Y. Yang, Y. M. Lu, and Y. Ran, Phys. Rev. B 84, 075129 (2011).

[8] G. B. Halász and L. Balents, Phys. Rev. B 85, 035103 (2012).

[9] H. Zhang, J. Wang, G. Xu, Y. Xu, and S.-C. Zhang, Phys. Rev. Lett. 112, 096804 (2014).

[10] J. Liu and D. Vanderbilt, Phys. Rev. B 90, 155316 (2014).

[11] H. Weng, C. Fang, Z. Fang, B. A. Bernevig, and X. Dai, Phys. Rev. X 5, 011029 (2015).

[12] S.-M. Huang, S.-Y. Xu, I. Belopolski, C.-C. Lee, G. Chang, B. Wang, N. Alidoust, G. Bian, M. Neupane, C. Zhang, S. Jia, A. Bansil, H. Lin, and M. Z. Hasan, Nat. Commun. 6, 7373 (2015).
[13] M. Hirayama, R. Okugawa, S. Ishibashi, S. Murakami, and T. Miyake, Phys. Rev. Lett. 114, 206401 (2015).

[14] A. A. Soluyanov, D. Gresch, Z. Wang, Q. Wu, M. Troyer, X. Dai, and B. A. Bernevig, Nature (London) 527, 495 (2015).

[15] J. Ruan, S.-K. Jian, H. Yao, H. Zhang, S.-C. Zhang, and D. Xing, Nat. Commun. 7, 11136 (2016).

[16] C. Fang, M. J. Gilbert, X. Dai, and B. A. Bernevig, Phys. Rev. Lett. 108, 266802 (2012).

[17] T. Guan, C. Lin, C. Yang, Y. Shi, C. Ren, Y. Li, H. Weng, X. Dai, Z. Fang, S. Yan, and P. Xiong, Phys. Rev. Lett. 115, 087002 (2015).

[18] S.-M. Huang, S.-Y. Xu, I. Belopolski, C.-C. Lee, G. Chang, T.-R. Chang, B. K. Wang, N. Alidoust, G. Bian, M. Neupane, D. Sanchez, H. Zheng, H.-T. Jeng, A. Bansil, T. Neupert, H. Lin, and M. Z. Hasan, PNAS 113, 1180 (2016).

[19] B. Q. Lv, H. M. Weng, B. B. Fu, X. P. Wang, H. Miao, J. Ma, P. Richard, X. C. Huang, L. X. Zhao, G. F. Chen, Z. Fang, X. Dai, T. Qian, and H. Ding, Phys. Rev. X 5, 031013 (2015).

[20] S.-Y. Xu, I. Belopolski, N. Alidoust, M. Neupane, G. Bian, C. Zhang, R. Sankar, G. Chang, Z. Yuan, C.-C. Lee, S.-M. Huang, H. Zheng, J. Ma, D. S. Sanchez, B. Wang, A. Bansil, F. Chou, P. 
P. Shibayev, H. Lin, S. Jia, and M. Z. Hasan, Science 349, 613 (2015).

[21] L. X. Yang, Z. K. Liu, Y. Sun, H. Peng, H. F. Yang, T. Zhang, B. Zhou, Y. Zhang, Y. F. Guo, M. Rahn, D. Prabhakaran, Z. Hussain, S. K. Mo, C. Felser, B. Yan, and Y. L. Chen, Nat. Phys. 11, 728 (2015).

[22] S.-Y. Xu, N. Alidoust, I. Belopolski, Z. Yuan, G. Bian, T.-R. Chang, H. Zheng, V. N. Strocov, D. S. Sanchez, G. Chang, C. Zhang, D. Mou, Y. Wu, L. Huang, C.-C. Lee, S.-M. Huang, B. Wang, A. Bansil, H.-T. Jeng, T. Neupert, A. Kaminski, H. Lin, S. Jia, and M. Zahid Hasan, Nat. Phys. 11, 748 (2015).

[23] B. Q. Lv, N. Xu, H. M. Weng, J. Z. Ma, P. Richard, X. C. Huang, L. X. Zhao, G. F. Chen, C. E. Matt, F. Bisti, V. N. Strocov, J. Mesot, Z. Fang, X. Dai, T. Qian, M. Shi, and H. Ding, Nat. Phys. 11, 724 (2015).

[24] H. J. Kim, K. S. Kim, J. F. Wang, M. Sasaki, N. Satoh, A. Ohnishi, M. Kitaura, M. Yang, and L. Li, Phys. Rev. Lett. 111, 246603 (2013).

[25] Q. Li, D. E. Kharzeev, C. Zhang, Y. Huang, I. Pletikosic, A. V. Fedorov, R. D. Zhong, J. A. Schneeloch, G. D. Gu, and T. Valla, Nat. Phys. (2016), doi:10.1038/nphys3648.

[26] X. C. Huang, L. X. Zhao, Y. J. Long, P. P. Wang, D. Chen, Z. H. Yang, H. Liang, M. Q. Xue, H. M. Weng, Z. Fang, X. Dai, and G. F. Chen, Phys. Rev. X 5, 031023 (2015).

[27] C.-L. Zhang, S.-Y. Xu, I. Belopolski, Z. Yuan, Z. Lin, B. Tong, G. Bian, N. Alidoust, C.-C. Lee, S.-M. Huang, T.-R. Chang, G. Chang, C.-H. Hsu, H.-T. Jeng, M. Neupane, D. S. Sanchez, H. Zheng, J. Wang, H. Lin, C. Zhang, H.-Z. Lu, S.-Q. Shen, T. Neupert, M. Z. Hasan, and S. Jia, Nat. Commun. 7, 10735 (2016).

[28] C. Shekhar, A. K. Nayak, Y. Sun, M. Schmidt, M. Nicklas, I. Leermakers, U. Zeitler, Y. Skourski, J. Wosnitza, Z. Liu, Y. Chen, W. Schnelle, H. Borrmann, Y. Grin, C. Felser, and B. Yan, Nat. Phys. 11, 645 (2015).

[29] J. Xiong, S. K. Kushwaha, T. Liang, J. W. Krizan, M. Hirschberger, W. Wang, R. Cava, and N. Ong, Science 350, 413 (2015).
[30] Z. Wang, Y. Zheng, Z. Shen, Y. Zhou, X. Yang, Y. Li, C. Feng, and Z.-A. Xu, Phys. Rev. B 93, 121112(R), (2016).

[31] C.-Z. Li, L.-X. Wang, H. Liu, J. Wang, Z.-M. Liao, and D.-P. Yu, Nat. Commun. 6, 10137 (2015).

[32] H. Li, H. T. He, H.-Z. Lu, H. C. Zhang, H. C. Liu, R. Ma, Z. Y. Fan, S.-Q. Shen, and J. N.Wang, Nat. Commun. 6, 10301 (2016).

[33] H. Z. Lu and S. Q. Shen, Phys. Rev. B 92, 035203 (2015).

[34] D. Xiao, M. C. Chang, and Q. Niu, Rev. Mod. Phys. 82, 1959 (2010).

[35] P. A. Lee and T. V. Ramakrishnan, Rev. Mod. Phys. 57, 287 (1985).

[36] See Supplemental Material at http://link.aps.org/supplemental/ 10.1103/PhysRevB.93.161110 for the detailed calculations.

[37] A. Altland and M. R. Zirnbauer, Phys. Rev. B 55, 1142 (1997).

[38] S. Hikami, A. I. Larkin, and Y. Nagaoka, Progr. Theor. Phys. 63, 707 (1980).

[39] N. H. Shon and T. Ando, J. Phys. Soc. Jpn. 67, 2421 (1998).

[40] E. McCann, K. Kechedzhi, V. I. Fal'ko, H. Suzuura, T. Ando, and B. L. Altshuler, Phys. Rev. Lett. 97, 146805 (2006).

[41] We only give the result for isotropic single-Weyl semimetals with $v_{F}=v_{z}=v_{\|}$; this simplification does not change any qualitative results with respect to quantum interference correction.

[42] We have carried out a coordinate transformation in deriving these results, where $\hbar k_{x}=\sqrt{k \sin \theta} \cos \varphi, \hbar k_{y}=$ $\sqrt{k \sin \theta} \sin \varphi, 2 \hbar m v k_{z}=k \cos \theta,-\mathbf{k}$ is obtained by setting $\varphi \rightarrow \varphi+\pi$ and $\theta \rightarrow \pi-\theta$.

[43] D. T. Son and B. Z. Spivak, Phys. Rev. B 88, 104412 (2013).

[44] A. A. Burkov, Phys. Rev. Lett. 113, 247203 (2014).

[45] S. B. Zhang, H. Z. Lu, and S. Q. Shen, arXiv:1509.02001.

[46] H. Suzuura and T. Ando, Phys. Rev. Lett. 89, 266603 (2002).

[47] F. V. Tikhonenko, A. A. Kozikov, A. K. Savchenko, and R. V. Gorbachev, Phys. Rev. Lett. 103, 226801 (2009).

[48] T. Ando, T. Nakanishi, and R. Saito, J. Phys. Soc. Jpn. 67, 2857 (1998).

[49] H.-Z. Lu and S.-Q. Shen, Phys. Rev. Lett. 112, 146601 (2014). 